\title{
The Italian Endo-COVID-19. Our response to the emergency
}

\author{
A. M. Isidori ${ }^{1}$ - E. A. Jannini ${ }^{2}$ A. Lenzi ${ }^{1}$ - E. Ghigo ${ }^{3}$ - the Board of Full Professors in Endocrinology, Directors of the \\ Endocrinology, Metabolic Disorders Residency Program
}

Received: 21 April 2020 / Accepted: 24 April 2020 / Published online: 8 May 2020

(C) Italian Society of Endocrinology (SIE) 2020

Dear Editor,

The Sars-COV-2 virus, a mutated coronavirus spreading from China in the last months of 2019, has a marked respiratory tropism, but in some subjects can causes an aberrant inflammatory response leading to a systemic disease for the simultaneous involvement of the lung, cardiovascular, coagulative, gastrointestinal and nervous system. Therefore an internist management is advised and many Endocrinologists and Endocrinology trainees have been called to help in those NHS hospitals facing the epidemic crisis.

Between 8 and 14 April 2020, the Directors of the Italian Endocrinology Residency Programs were asked to fill in a survey on the number of hospital beds, physicians, residents, nurses and technicians were made available by the School for NHS reorganization during phase one of lockdown (7 March-7 April 2020). All agreed with 100\% completion rate, for a total of 34 Programs, expression of 35 Universities and 45 Hospitals. The data are reported in the Table as cumulative national and stratified for macroregional areas (North, Middle, South and islands) because of different viral spread (Table 1).

In $22.9 \%$ of cases, the whole Endocrine Department was converted to hold COVID-19 patients $(36.8 \%$ in the North). At least half of the ordinary beds were given to the emergency by $41.7 \%$ of centres nationwide, $68.4 \%$ in the

\footnotetext{
A. M. Isidori

andrea.isidori@uniroma1.it

E. Ghigo

ezio.ghigo@unito.it

1 Dipartimento di Medicina Sperimentale, Sapienza Università di Roma, Policlinico Umberto I - COVID Hospital, Rome, Italy

2 Dipartimento di Medicina dei Sistemi, Università di Roma Tor Vergata, Rome, Italy

3 Dipartimento di Scienze Mediche, Università di Torino, Turin, Italy
}

North, and $23-25 \%$ in the rest of Italy. Overall, $45.8 \%$ of the Departments maintained their own endocrine beds.

Regarding the specialty physicians (endocrinologists of the NHS), in $15.8 \%$ of northern Italy the entire staff was dedicated to handle COVID-19 patients, whereas it dropped to $6.3 \%$ nationwide. However, in $66.2 \%$ of sites of North and Middle, at least half of specialists have been used for the emergency. This means that 168 endocrinologists (out of 502) have dedicated most of their working hours to COVID-19 related activities (141 in North, 31 in Middle and 9 in South Italy). Similarly occurred for nurses usually working in Endocrinology or Diabetology. Overall 192 nurses have dedicated most of their time to COVID-19 healthcare. The reorganization resulted in a reduction or suspension of the Endocrine Day-Hospital admissions for $89.6 \%$ of sites, but outpatient activities, limited to urgent and non-deferrable outpatients, was almost universally guaranteed.

Coming to the contribution of the residents in Endocrinology, a remarkable help was given to crisis handling. A third of the Schools have made available more than half of their own trainees $(31.2 \%)$, and an additional $16.7 \%$ to a lower proportion. Translated into numbers, it corresponds to a "small army" of at least 190 young in-training endocrinologists who are helping COVID-19 wards. The involvement, was much higher in the North (in $68.4 \%$ more than half of the trainees were used), smaller in the Middle (12.6\%), not requested in the South of Italy. The latter finding must be interpreted in light of the different geographical impact that the pandemic. An important aspect is that adhesion to the COVID-19 emergency was voluntary in $41.7 \%$ of cases of medical doctors.

In summary, this survey demonstrates the ability and readiness of endocrinology-in all its subspecialties diabetes, metabolism and andrology - to support our NHS bringing competence, dedication and sense of responsibility. Our response to COVID-19 is not only the best handling 
Table 1 The response of the Endocrinology Residency Programs to the COVID-19 epidemic

\begin{tabular}{|c|c|c|c|c|}
\hline & Total & North & Middle & South/Islands \\
\hline Schools $(n)$ & 34 & 16 & 11 & 7 \\
\hline NHS Endocrinologist employed in the Programs $(n)$ & 502 & 223 & 163 & 116 \\
\hline Trainees in the 4-year Endocrinology Programs $(n)$ & 403 & 166 & 145 & 92 \\
\hline NHS Nurses/technicians $(n)$ & 543 & 247 & 174 & 122 \\
\hline \multicolumn{5}{|l|}{ Ordinary beds converted to hold COVID-19 patients (\%) } \\
\hline 0 & 45.8 & 26.3 & 62.4 & 53.8 \\
\hline $1-50$ & 12.5 & 5.3 & 12.5 & 23.1 \\
\hline $51-99$ & 18.8 & 31.6 & 18.8 & 0 \\
\hline 100 & 22.9 & 36.8 & 6.3 & 23.1 \\
\hline \multicolumn{5}{|l|}{ Endocrinologists dedicated $*$ to COVID-19 wards (\%) } \\
\hline 0 & 37.5 & 10.5 & 43.7 & 69.2 \\
\hline $1-50$ & 29.2 & 26.3 & 37.5 & 23.1 \\
\hline $51-99$ & 27.1 & 47.4 & 18.8 & 7.7 \\
\hline 100 & 6.3 & 15.8 & 0 & 0 \\
\hline \multicolumn{5}{|l|}{ Nurses dedicated $*$ to COVID-19 services (\%) } \\
\hline 0 & 35.4 & 21.1 & 50 & 38.5 \\
\hline $1-50$ & 29.2 & 26.3 & 25 & 38.5 \\
\hline $51-99$ & 22.9 & 31.6 & 25 & 7.7 \\
\hline 100 & 12.5 & 21.1 & 0 & 15.3 \\
\hline \multicolumn{5}{|l|}{ Trainees dedicated $*$ to COVID-19 patients (\%) } \\
\hline 0 & 52.1 & 5.3 & 68.8 & 100 \\
\hline $1-50$ & 16.7 & 26.3 & 18.8 & 0 \\
\hline $51-99$ & 16.7 & 36.8 & 6.3 & 0 \\
\hline 100 & 14.5 & 31.6 & 6.3 & 0 \\
\hline \multicolumn{5}{|l|}{ Medical doctors transfer occurred (\%): } \\
\hline Mandatorily & 27.1 & 21.1 & 31.0 & 30.8 \\
\hline Voluntarily & 41.7 & 57.9 & 43.8 & 15.4 \\
\hline No transfer occurred & 12.5 & 0 & 6.3 & 38.4 \\
\hline Other & 18.7 & 21 & 18.9 & 15.4 \\
\hline
\end{tabular}

$*>50 \%$ working hours of endocrine patients [1-5] but also a large scale human resources mobilization that should be a source of pride for all Endocrinology Programs, Directors, Coordinators and all physicians and trainees who dedicate their lives to science and healthcare of our country.

Acknowledgement We are grateful to Dr. Carlotta Pozza for creating the web form and contributing to data analyses. We dedicate this letter to all Medical Doctors who have contracted COVID-19 while on duty and suffered or lost their lives because of it.

Author contributions All authors contributed to the drafting and data analysis.

Funding No funding was used to produce this manuscript.

\section{Compliance with ethical standards}

Conflict of interest Nothing to disclose related to this work.

Ethics approval This research involves human participants, but no sensitive data were collected. The protocol was approved by the local board of the Department of Experimental Medicine - Sapienza University.

Informed Consent Consent to participate is not required.

\section{References}

1. Fadini GP, Morieri ML, Longato E, Avogaro A (2020) Prevalence and impact of diabetes among people infected with SARS-CoV-2. J Endocrinol Invest. https://doi.org/10.1007/s40618-020-01236-2 
2. Scaroni C, Armigliato M, Cannavo S (2020) COVID-19 outbreak and steroids administration: are patients treated for Sars-Cov-2 at risk of adrenal insufficiency? J Endocrinol Invest. https://doi. org/10.1007/s40618-020-01253-1

3. Trimarchi F (2020) An invisible hand has guided clinical endocrinology toward an unexpected future. J Endocrinol Invest. https:// doi.org/10.1007/s40618-020-01256-y

4. Isidori AM, Arnaldi G, Boscaro M, Falorni A, GIordano C, Giordano R, Pivonello R, Pofi R, Hasenmajer V, Venneri MA, Sbardella E, Simeoli C, Scaroni C, Lenzi A (2020) COVID-19 infection and glucocorticoids: update from the Italian Society of Endocrinology Expert Opinion on steroid replacement in adrenal insufficiency. J Endocrinol Invest. 2020. https://doi.org/10.1007/ s40618-020-01266-w
5. Paoli D, Pallotti F, Colangelo S, Basilico F, Mazzuti L, Turriziani O, Antonelli G, Lenzi A, Lombardo F (2020) Study of SARS$\mathrm{CoV}-2$ in semen and urine samples of a volunteer with positive rino-pharyngeal swab. J Endocrinol Invest. 2020. https://doi. org/10.1007/s40618-020-01261-1

Publisher's Note Springer Nature remains neutral with regard to jurisdictional claims in published maps and institutional affiliations. 